\title{
Identification of Damping Ratio for a Rotor-Bearing System Using a Magnetic Bearing Exciter
}

\author{
Yongjiang Zhu ${ }^{1, a}$, Ya Zhang ${ }^{2, b}$, Weimin Wang ${ }^{3, c}$, Jianfei Yao ${ }^{4, d}$ \\ ${ }^{1,2,3,4}$ Diagnosis and Self-recovery Engineering Research Center, Beijing University of Chemical \\ Technology, Beijing 100029, China \\ azyj319205@126.com, bzhangya@mail. buct.edu. cn, cwwmbuct@163.com, \\ dyaojf@mail.buct.edu.cn
}

Keywords: magnetic bearing exciter, an asynchronously rotating force, damping ratio, half-power point method

\begin{abstract}
A magnetic bearing exciter is attached to the rotor-bearing system, to exert an asynchronously rotating force on the rotor to excite the first forward mode. And the half-power point method is developed on the basis of displacement response function, where displacement response data is needed only to identify the frequency and damping. The simulation results show that the method can effectively identify the damping with speed and white noise interference. The experiment results show that the damping radio decreases with increasing rotational speed. It has a good engineering application prospects.
\end{abstract}

\section{Introduction}

Centrifugal compressor instability remains a major concern causing reduced unit availability and project commissioning delays, leading to revenue lost for both users and vendors of the equipment. The evaluation of rotor stability has become an essential part of rotordynamic analyses and rotating machinery design. The damping ratio is an important index to use when evaluating stability of the rotor system. It is defined by the API Standard 617 [1] as "Stability analysis is the determination of the natural frequencies and the corresponding logarithmic decrements of the damped rotor/support system using a complex eigenvalue analysis."

To determine the damping of the damped rotor/support system under operational conditions, many researches have been done. Multiple output backward autoregression (MOBAR) method was proposed by C. Hunter Cloud [2] for estimating the damping ratio of complex systems. When stability measurements were performed by the Dresser-Rand [3], Sulzer Turbo Ltd [4,5] and Hitachi Plant Technologies Ltd[6] , a magnetic bearing exciter was used. Particularly, extensive stability measurements were performed in Sulzer Turbo Ltd [4], the damping ratio was evaluated by the circle fit method and good results had been achieved.

In this paper, a magnetic bearing exciter is attached to the rotor-bearing system, to inject an asynchronously rotating force into the rotor to excite the first forward precession mode. And we are developing the half-power point method on the basis of displacement response function, where displacement response data is needed only to identify the frequency and damping. And we conducted a theoretical derivation and the simulation using this method. Finally, to research the relationship between rotational speed and damping, the damping test on the bedstand at various speeds. Experimental study demonstrates, the damping radio decreases with increasing of rotating speed in a rotor-bearing system.

\section{Theoretical derivation}

In general, consider a single-degree-of-freedom system subject to an excitation, the differential equation of motion is:

$$
m \ddot{x}(t)+c \dot{x}(t)+k x(t)=f(t)
$$


where $\mathrm{m}$ is known as the mass, $\mathrm{c}$ is the damping and $\mathrm{k}$ is the stiffness. $\mathrm{x}(\mathrm{t})$ is the vibration displacement of SDOF system and $\mathrm{f}(\mathrm{x})$ is an excitation.

Fourier transform to Eq. (1), it can be obtained:

$$
\left(\omega_{0}^{2}-\omega^{2}+2 j \xi \omega_{0} \omega\right) x(\omega)=\frac{F(\omega)}{m}
$$

where

$$
x(t)=A \sin (\omega t-\varphi)
$$

and

$$
\xi=\frac{c}{2 m \omega_{0}}
$$

$\omega_{0}$ is called the undamped natural frequency (radians per second), $\xi$ is a nondimensional parameter, called damping ratio or damping factor.

Therefore, the displacement frequency response function of SDOF system can be expressed as:

$$
H_{d}(\omega)=\frac{x(\omega)}{F(\omega)}=\frac{1}{m\left(\omega_{0}^{2}-\omega^{2}+2 j \xi \omega_{0} \omega\right)}
$$

And the magnitude of displacement frequency response function can be written as:

$$
\frac{F_{0}}{m \sqrt{\left(\omega_{0}^{2}-\omega^{2}\right)^{2}+\left(2 \xi \omega_{0} \omega\right)^{2}}}=\frac{A_{\max }}{\sqrt{2}}=\frac{1}{\sqrt{2}} \frac{F_{0}}{2 m \xi \omega_{0}^{2} \sqrt{1-\xi^{2}}}
$$

It reflects the relationship between the amplitude of displacement frequency response function and the frequency $\omega$ of the excitation applied to SDOF system.

In Eq.(1), take $f(t)=F_{0} \sin \omega t$ to represent a frequency-sweep force, $F_{0}$ is the excitation amplitude, the frequency $\omega$ of excitation gradual changes in the frequency domain containing the system resonance frequency band. We can obtain the following equations:

$$
\ddot{x}(t)+2 \xi \omega_{0} \dot{x}(t)+\omega_{0}^{2} x(t)=\frac{F_{0}}{m} \sin \omega t
$$

The special solution of Eq. (7) can be written in the form:

$$
x(t)=A \sin (\omega t-\varphi)
$$

where

$$
A=\frac{F_{0}}{m \sqrt{\left(\omega_{0}^{2}-\omega^{2}\right)^{2}+\left(2 \xi \omega_{0} \omega\right)^{2}}}
$$

and

$$
\varphi=\tan ^{-1} \frac{2 \xi \omega_{0} \omega}{\omega_{0}^{2}-\omega^{2}}
$$

In Eq. (9), it reflects the relation between the amplitude of displacement response function and the frequency $\omega$ of the excitation.

Compare Eq. (6) and Eq. (9), the following holds:

$$
A=F_{0}\left|H_{d}(\omega)\right|
$$


where $F_{0}$ is a constant. Therefore, within the frequency domain $\omega$ of frequency-sweep force, the frequency in the half-power point on the amplitude-frequency response curve of displacement response function is equal to that in the half-power point on the amplitude-frequency curve of displacement frequency response function. That is, the damping ratio can be calculated by using the half-power point method on the amplitude-frequency response curve of displacement response function. Eq. (9) expressed the displacement response function of frequency response curve shown in Figure 1.

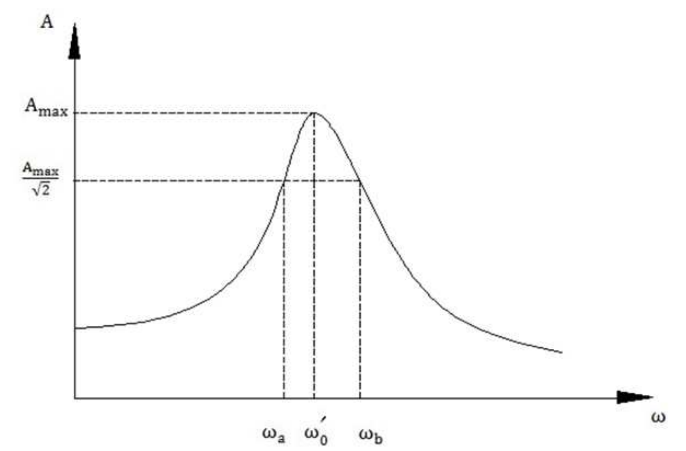

Fig. 1. The amplitude-frequency response curve of displacement response function

The frequency $\omega_{0}^{\prime}$ corresponding to the peak of amplitude-frequency response curve, can be calculated by the method of extremum in Eq.(9). The following is obtained:

$$
\omega_{0}^{\prime}=\omega=\omega_{0} \sqrt{1-2 \xi^{2}}
$$

When $\xi \leq 0.1$, it can be considered that $\omega_{0}^{\prime} \approx \omega_{0}$.

In the half-power point, the magnitude of the displacement response function can be expressed as:

$$
\frac{F_{0}}{m \sqrt{\left(\omega_{0}^{2}-\omega^{2}\right)^{2}+\left(2 \xi \omega_{0} \omega\right)^{2}}}=\frac{A_{\max }}{\sqrt{2}}=\frac{1}{\sqrt{2}} \frac{F_{0}}{2 m \xi \omega_{0}^{2} \sqrt{1-\xi^{2}}}
$$

The solution can be obtained:

$$
\begin{aligned}
& \omega_{\mathrm{a}} \approx \omega_{0}(1-\xi) \\
& \omega_{\mathrm{b}} \approx \omega_{0}(1+\xi)
\end{aligned}
$$

So, it can be got:

$$
\xi=\frac{\omega_{b}-\omega_{a}}{2 \omega_{0}}
$$

\section{Simulation}

Considering a single-disc rotor system. Assuming the shaft is massless, and take the mass of rigid disc $\mathrm{m}=25 \mathrm{~kg}$, viscous damping $\mathrm{c}=5000 \mathrm{~N} \cdot \mathrm{m} / \mathrm{s}$, stiffness $\mathrm{k}=6.3 \times 10^{6} \mathrm{~N} / \mathrm{m}$. So, the natural frequency $\mathrm{f}_{0}=80 \mathrm{HZ}$ and damping ratio $\xi=19.9 \%$. And the rotational speed is $2400 \mathrm{rpm}$. Apply a frequency-sweep force (the frequency range from $20 \mathrm{HZ}$ to $120 \mathrm{HZ}$ ) to the system with white noise or not add white noise interference. 
The time signal of vibration response to be obtained, and then fast Fourier transformed(FFT). Within the sweep frequency range, the vibration response caused by the excitation is extracted in frequency domain. The amplitude-frequency response curve of displacement response function can be plotted and fitted by the least square method, shown in Figure 2.

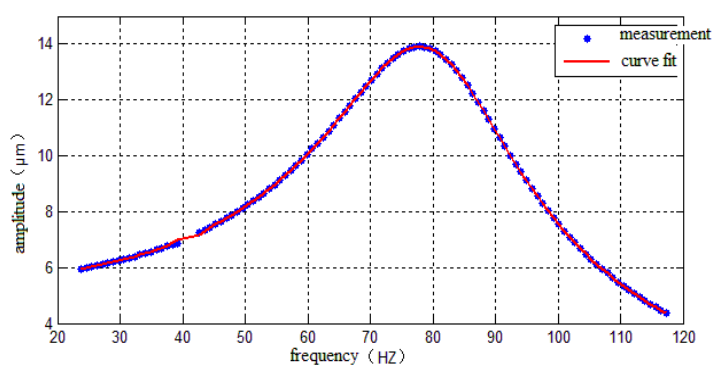

(a) with white noise

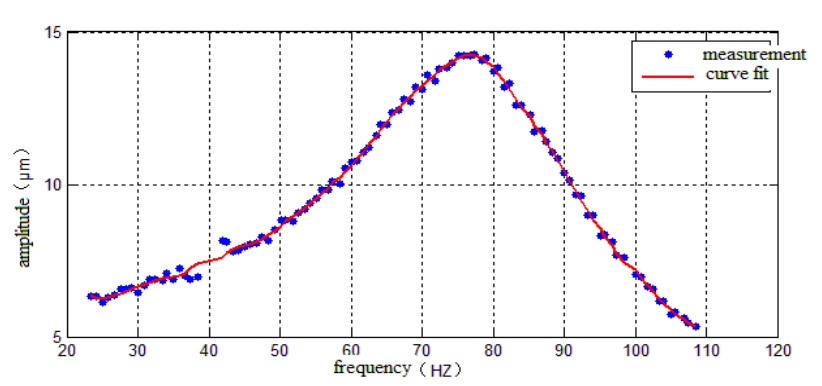

(b) without white noise

Fig. 2. The amplitude-frequency response curve of displacement response function

By the half-power point method, it can be obtained: with white noise, $\mathrm{f}_{0}=79.78 \mathrm{HZ}, \xi=20.32 \%$; without white noise, $\mathrm{f}_{0}=78.61 \mathrm{HZ}, \xi=20.47 \%$. By contrast, we can get the conclusion that this method can effectively identify the damping with speed and white noise interference.

\section{Experimental Research}

Test Procedure. Test bed structure is shown in Figure 3. To research the relation between rotational speed and damping, the damping test on the bedstand at 1500rpm, 1800rpm, 2100rpm, 2400rpm, 2700rpm, 3000rpm, 3300rpm. In the test, the first natural frequency is excited asynchronously. A magnetic bearing exciter is attached to the rotor-bearing system, to inject an asynchronously rotating force into the rotor to excite the first forward precession mode. The shaft response is measured while running the magnetic force excitation frequency through the natural frequency of the rotor, to extract the amplitude-frequency response data of displacement response function.
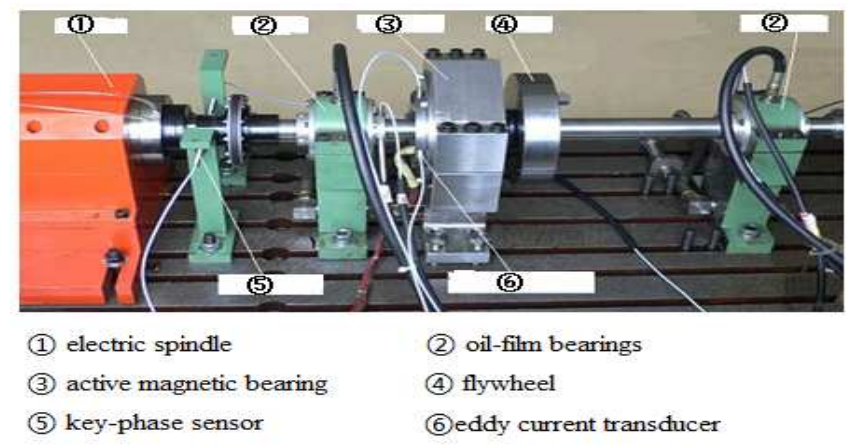

Fig. 3. Photograph of experimental system

As shown in Figure 4 the damping ratio measurements are carried out by applying a harmonic force with a sweep over a frequency range from $10 \mathrm{~Hz}$ to $110 \mathrm{~Hz}$. To avoid any distortion of the measured resonance curves a frequency increase rate of $1 \mathrm{~Hz} / \mathrm{sec}$ is defined. The level of the excitation force was adjusted in order to ensure high quality responses. 


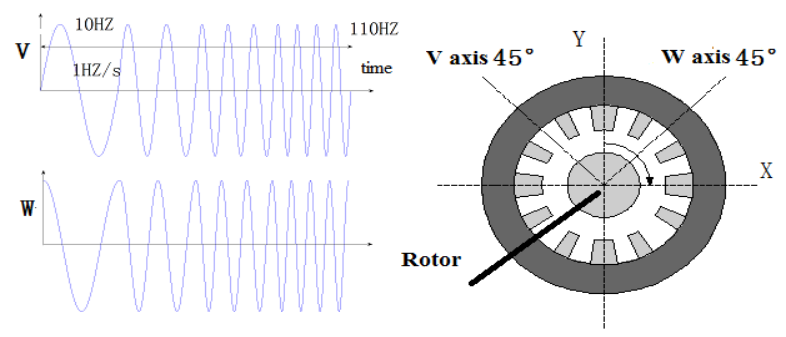

Fig. 4. Power Signal of the Magnetic Bearing Exciter

Test Results. Figure 5 shows waterfall plots of the rotor while exciting the forward precession mode. It can clearly be seen that the rotor speed remains constant while the injection force sweeps through the frequency range resulting in a peak response at the first rotor natural frequency. The results of the evaluation are visualized in the amplitude-frequency response curve of displacement response function. Figure 6 shows an amplitude-frequency response curve for the originally measured data and the curve fit superimposed. The damping ratio is determined by fitting the best curve through the plotted points for each resonance with the half power point band. The results of damping ratio measurements, Figure 7 , show that the stability of the rotor system measured has change at different speeds. The most remarkable result on the measured damping curves is, the damping radio decreases with increasing of rotating speed. It is the same as expected. As rotational speed increases, the swirling fluid in oil-film bearings gaps leads to cross-coupled stiffness growth, which reduces the damping of the forward mode.

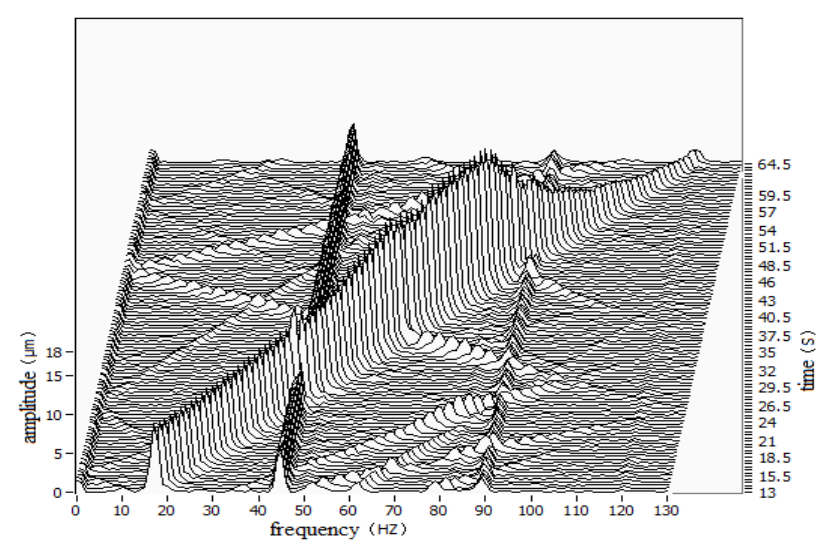

Fig. 5. Waterfall Plot of test process at 2700rpm

\section{Conclusions}

(1)The method to identify damping is proposed. Displacement response data is needed only to identify the frequency and damping ratio while running the magnetic force excitation frequency through the natural frequency of the rotor.

(2) The simulation results show that the method can effectively identify the damping with speed and white noise interference.

(3) The experiment results show that the damping ratio decreases with increasing of rotating speed.

(4)This method is simple, reliable, and suitable for damping ratio measurement of rotating machinery under operational conditions. So, it has a good engineering application prospects. 


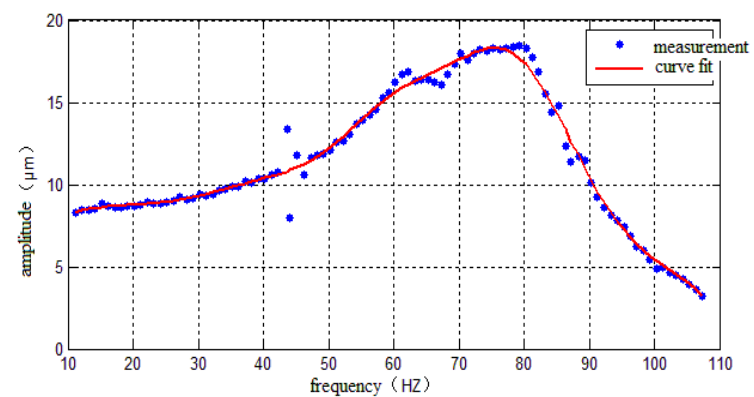

Fig. 6. The amplitude-frequency response curve of displacement response function at 2700rpm
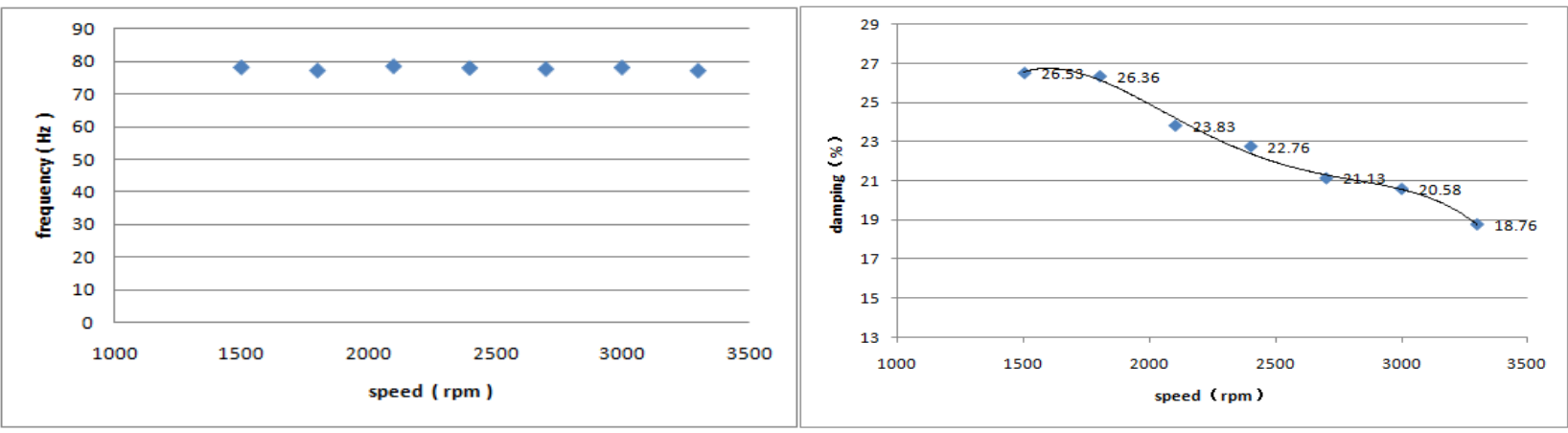

Fig. 7. Measured frequency and damping ratio

\section{Acknowledgments}

This work was supported by the general Program of Natural Science Foundation of China (50975018)

\section{References}

[1] API Standard 617, Axial and Centrifugal Compressors and Expander-Compressors for Petroleum, Chemical and Gas Industry Services, Seventh Edition, American Petroleum Institute, Washington, D.C, 2003.

[2] C.Hunter Cloud, Eric H.Maslen, Lloyd E.Barrent. Damping Ratio Estimation Techniques for Rotordynamic Stability Measurements, J. Journal of Engineering for Gas Turbines and Power, 2009.1, Vol.131/012504-11.

[3] Thomas A.Soulas, Mark J.Kuzdzal. Rotordynamic testing and evaluation of alarge centrifugal compressor using a magnetic bearing exciter. Proceedings of ASME Turbo Expo 2009: Power for Land,Sea and Air,GT2009-60320.

[4] Urs Baumann. Rotordynamic stability tests on high-pressure radial compressors. Proceedings of 28th Turbomachinery Symposium. Copyright 1999.

[5] Yves Bidaut, Urs Baumann, Salim, Mohamed Hamed Al-Harthy. Rotordynamic stability of a 9500 PSI reinjection centrifugal compressor equipped with a hole pattern seal-measurementversus prediction taking into account the operational boundary conditions. Proceedings of the thirty-elghth turbomachinery symposium , 2009.

[6] Hiroyuki Fujiwara, Yasuo Fukushima, Naohiko Takahashi, Hiroto Oyama, Osami Matsushita. Evaluation of Q-values of a rotor-bearing system using a modal open loop transfer function. Proceeding of ASME Turbo Expo2009: Power for Land, Sea and Air. GT2009-5 